\title{
Sistema biológico alternativo para pós-tratamento de esgoto
}

\section{ALTERNATIVE BIOLOGICAL SYSTEM AS A WASTEWATER POS-TREATMENT}

\author{
Priscila Karina Altvater Mannich(1) \\ Daniel Costa dos Santos $\left({ }^{2}\right)$ \\ Michael Mannich( ${ }^{(3)}$
}

(1)Engenheira Ambiental. Mestre em Engenharia de Recursos Hídricos e Ambiental pela Universidade Federal do Paraná (UFPR).

(2)Engenheiro Civil. Mestre em Engenharia Civil pela Universidade Federal do Rio Grande do Sul. Doutor em Engenharia Civil pela Universidade de São Paulo. Professor do Departamento de Hidráulica e Saneamento da Universidade Federal do Paraná (UFPR).

() ${ }^{3}$ Engenheiro Ambiental. Mestre em Engenharia de Recursos Hídricos e Ambiental pela Universidade Federal do Paraná. Doutorando em Engenharia de Recursos Hídricos e Ambiental pela Universidade Federal do Paraná (UFPR).

Endereço: Universidade Federal do Paraná, Departamento de Hidráulica e Saneamento - Caixa Postal 19011 - Cep: 81531-990 Curitiba -PR.e-mail: paltvater@yahoo.com.br Entrada:13/4/2009 Aprovação: 25/6/2009

\section{RESUMO}

A situação crítica de tratamento de esgoto doméstico no país requer alternativas para evitar a poluição hídrica. O objetivo deste trabalho foi avaliar a eficiência do pós-tratamento de efluente de Tanques Sépticos (TS) num sistema inovador denominado Unidade Tubular de Tratamento de Esgoto (UTTE), conjuntamente com modificações do TS. O UTTE consiste de uma tubulação a qual é preenchida com um material suporte para a aderência e crescimento da biomassa. o princípio de remoção da matéria orgânica é predominantemente anaeróbio e com características de um biofiltro. O material suporte para promover o crescimento da biomassa foi confeccionado a partir de tiras de garrafa PET previamente lixadas. O UTTE se apresentou como um bom sistema de pós-tratamento para o TS, com eficiências médias de remoção para o sistema TS-UTTE de 66\%, 70\% e 74\% para DBO, DQO e SS, respectivamente.

\section{ABSTRACT}

The critical situation of domestic wastewater treatment in Brazil requires alternatives to prevent water pollution. The purpose of this study was to evaluate the efficiency of post-treatment of sewage from septic tanks in a reactor denominated tubular treatment of the domestic effluent (UTTE), together with changes in the septic tank. The UTTE consist of a pipe which is filled with a material support for the adhesion and growth of biomass. The principle of removing organic matter is predominantly anaerobic and with characteristics of a biofilter. The PVC pipe was filled with a support media to promote the development of biomass and therefore the treatment of effluent. For this support media it was used strips of PET.. The UTTE behaved as a good post treatment to the septic tank, with a removal of the system TS-UTTE of $66 \%, 70 \%$ and $74 \%$ for BOD, COD and SS, respectively.

Palavras-chave: tratamento de esgoto; tanque séptico, biofilme; meio suporte.

Key-Words: wastewater treatment, septic tank, biofilm, support media

\section{1 - INTRODUÇÃO}

Nos últimos anos, a crescente preocupação com a qualidade do meio ambiente e o avanço de tecnologias para o tratamento de efluentes líquidos, melhoraram a situação nacional quanto ao tratamento de esgoto doméstico. Todavia, os índices de tratamento e a eficiência dos processos estão aquém do desejável. Dada a situação econômica nacional e de muitos outros países, o uso de sistema de baixo custo torna-se fundamental. O sistema de tanques sépticos (TS) para tratamento de esgotos é o mais usado em todos os países devido à sua simplicidade de construção e operação, além de baixo custo de implementação. Apesar de todas as vantagens, a eficiência do tanque séptico para remoção de matéria orgânica é moderada, necessitando de um pós-tratamento para alcançar um grau de remoção da matéria orgânica aceitável.

No Paraná, dos 748 distritos existentes, 592 não possuem rede coletora de esgoto e destes, 238 distritos utilizam tanques sépticos (TS) como destino final do esgoto. (IBGE, 2002). Segundo o SNIS (2003) 50\% dos domicílios são conectadas à rede de coleta de esgoto, mas apenas $27 \%$ do esgoto gerado é tratado, um índice 
lamentavelmente baixo. A população que não dispõe de sistema de tratamento de esgoto tem seus efluentes lançados in natura nos cursos d'água, além das ligações irregulares, com rede de esgoto conectada a galerias pluviais, sendo esta, uma das principais causas da poluição dos corpos d'água no Brasil. Assim, procura-se desenvolver tecnologias para tratamento de águas residuárias que sejam mais eficientes e com o menor custo possível, reduzindo consumo de energia e de substâncias químicas, além de possibilitar o reuso da água.

Em diversos países ainda há um preconceito contra sistemas naturais por não incluir equipamentos mecânicos, implicitamente considerados por alguns como garantia de eficiência. O tratamento anaeróbio de águas residuárias tem sido estudado, principalmente como uma alternativa de tratamento de baixo consumo de energia e custo operacional, em substituição aos processos de custos mais elevados, como o sistema de lodos ativados ou ainda, para diminuir áreas destinadas ao tratamento por sistemas de lagoas. No Brasil, por exemplo, o sistema de lagoas de estabilização está totalmente estabelecido e parece haver pouco preconceito contra o mesmo (ANDRADE NETO, 1993).

Neste contexto, considerando a importância do desenvolvimento de tecnologias sustentáveis para o tratamento de esgoto, o foco da pesquisa é avaliar o póstratamento de efluentes de tanques sépticos através de um novo sistema denominado Unidade de Tratamento Tubular de Esgoto (UTTE).

\section{2 - MATERIAIS E MÉTODOS}

Nesse trabalho foi projetado e operado um sistema em escala de bancada (semi-piloto), composto de um Tanque Séptico (TS) modificado por meio da inserção de cortinas para desenvolvimento de biomassa, seguido de uma Unidade Tubular de Tratamento de Esgoto (UTTE) para o pós-tratamento do efluente do TS. Durante a operação foi utilizado um efluente sintético, o qual representa características físico-químicas semelhantes às do esgoto doméstico. O sistema foi instalado na Universidade Federal do Paraná, no Campus Centro Politécnico, em área anexa ao Departamento de Hidráulica e Saneamento. As análises do monitoramento do esgoto sintético bruto e tratado em cada uma das unidades foram realizadas no laboratório de Engenharia Ambiental. A Figura 1 ilustra o fluxograma do sistema de tratamento e os locais onde foram coletadas as amostras (P1, P2 e P3).

O esgoto sintético é armazenado num tanque de armazenamento de esgoto (TA), a partir do qual é ele-
TS

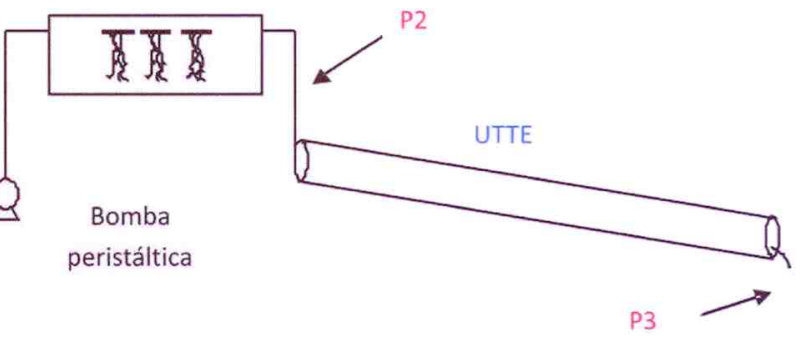

Figura 1 - Sistema TS-STTE

vado até o tanque séptico (TS) por meio de uma bomba peristáltica, e que por sua vez atinge a unidade de tratamento tubular de esgoto (UTTE) por gravidade.

\subsection{O Esgoto Sintético}

O sistema foi operado com esgoto sintético por 3 razões: (i) segurança sanitária; (ii) dificuldade de canalização de esgoto até o local e; (iii) padronização do efluente. A composição do despejo sintético utilizado foi a formulação apresentada por Torres (1992) (Tabela 1). O esgoto sintético produzido foi armazenado por um período de 4 dias, num tanque de armazenamento (TA) com capacidade de 160 litros, mantido sob condições naturais, sendo que após esse período o esgoto sintético não utilizado era descartado e substituído.

\begin{tabular}{|c|c|}
\hline Constituintes & $1 \mathrm{~L}$ \\
\hline \multicolumn{2}{|c|}{ COMPOSTO ORGÂNICO } \\
\hline Extrato de Carne (g) & 0,2 \\
\hline Amido (g) & 0,01 \\
\hline Farinha de trigo $(\mathrm{g})$ & 0,0 \\
\hline Sacarose $(\mathrm{g})$ & 0,0175 \\
\hline $\mathrm{NH}_{4} \mathrm{Cl}(\mathrm{g})$ & 0,006375 \\
\hline Óleo $(\mathrm{mL})$ & 0,051 \\
\hline Detergente (gotas) & 2 \\
\hline Celulose (g) & 0,03 \\
\hline \multicolumn{2}{|c|}{ SAIS MINERAIS } \\
\hline $\mathrm{NaCl}(\mathrm{g})$ & 0,25 \\
\hline $\mathrm{MgCl}_{2} 6 \mathrm{H}_{2} \mathrm{O}(\mathrm{g})$ & 0,0007 \\
\hline $\mathrm{Ca} \mathrm{Cl}_{2}, 2 \mathrm{H}_{2} \mathrm{O}(\mathrm{g})$ & 0,0045 \\
\hline $\mathrm{KH}_{2} \mathrm{PO}_{4}(\mathrm{~g})$ & 0,0264 \\
\hline \multicolumn{2}{|c|}{ SOLUÇÃO TAMPÃO } \\
\hline Bicarbonato de Sódio $(\mathrm{g})$ & 0,2 \\
\hline
\end{tabular}

Fonte: Torres (1992)

\section{Tabela 1 - Composição do esgoto sintético}

No TA foi instalado um misturador lento que operou ininterruptamente promovendo a constante mistura do despejo sintético a fim de homogeneizar o efluente e reduzir a sedimentação dos sólidos no tanque. Deste modo esperou-se reduzir as variações na concentração de entrada do tanque séptico. 


\subsection{O Tanque Séptico}

O Tanque Séptico, do tipo câmara única, foi projetado em escala reduzida por BARBOSA (2006) segundo recomendações da NBR-7229/ABNT (1993). Na unidade foi instalado um sistema que permite variar a relação de forma comprimento/largura (C/L) de $4 / 1$ a 6/1 (Figura 4.3), a fim de avaliar relações diferentes daquelas recomendadas pela NBR 7229/ABNT (1993) cujos resultados são discutidos por ALTVATER (2008) e também em futura publicação. Foram também instalados ganchos no tampo superior da unidade TS para permitir a fixação das cortinas para meio aderido.

O TS, ilustrado na Figura 2, foi construído em estrutura de acrílico transparente com $120 \mathrm{~cm}$ de comprimento, $20 \mathrm{~cm}$ de largura e $17 \mathrm{~cm}$ de altura. No interior do TS foram instaladas duas estruturas, uma a $80 \mathrm{~cm}$ e outra a $100 \mathrm{~cm}$ da entrada no sentido longitudinal, para que pudesse ser alterada a relação de forma. Para este estudo, as dimensões longitudinal e transversal foram, respectivamente, $80 \mathrm{~cm} \mathrm{e} 20 \mathrm{~cm}$, o que equivale a relação de forma C/L igual a $4 / 1$ e um volume útil de 27,2 litros. Os anteparos foram instalados a $5 \mathrm{~cm}$ da entrada e a $5 \mathrm{~cm}$ da saída.

Como a unidade TS, construída em acrílico, foi instalada em local exposto, foi necessário revestir a unidade com papel alumínio, para impedir a penetração da luz, evitando a proliferação de algas e proporcionando uma simulação das condições mais próximas da sua real condição de operação.

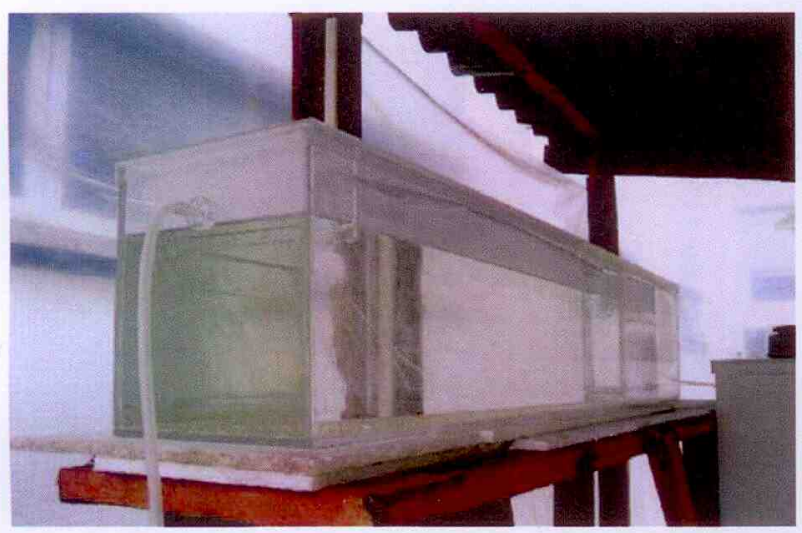

Figura 2 - tanque séptico experimental

O TS foi modificado através da inserção de cortinas que proporcionam um suporte fixo para desenvolvimento de biomassa. O objetivo é aumentar a eficiência de remoção da matéria orgânica solúvel no despejo, sem comprometer o processo de sedimentação de sólidos e digestão do lodo. As cortinas foram confeccionadas utilizando material de garrafas PET, as quais foram cortadas, lixadas e agrupadas em 6 grupos de tiras em forma cilíndrica e dispostas em separado a fim de não atrapalhar o fluxo do escoamento. O lixamento foi feito no sentido longitudinal, com lixa para madeira. Cada cortina possui 17 tiras com 1,5 cm de largura e 15,5 cm de altura, perfazendo um total de $790,5 \mathrm{~cm}^{2}$ por cortina e $0,47 \mathrm{~m}^{2}$ de área de contato total para as 6 cortinas inseridas no TS, com uma área específica de 17,6 m2/m3 do TS. A Figura 4 ilustra as cortinas e a Figura 5 a disposição destas no TS após um período de operação, as quais foram organizadas em 2 linhas de 3, paralelas ao sentido longitudinal.

A escolha das garrafas PET para confecção das cortinas foi motivada sob uma ótica ambiental do aproveitamento de um material muitas vezes descartado em abundância e que poderia ser reciclado ou reutilizado. E sob ponto de vista técnico, ALTVATER (2005) demonstrou em estudo experimental em rede de drenagem urbana que as tiras de garrafas PET apresentaram satisfatório crescimento de biomassa e resistência, além de ser um material inerte e, portanto, adequado ao tratamento biológico.

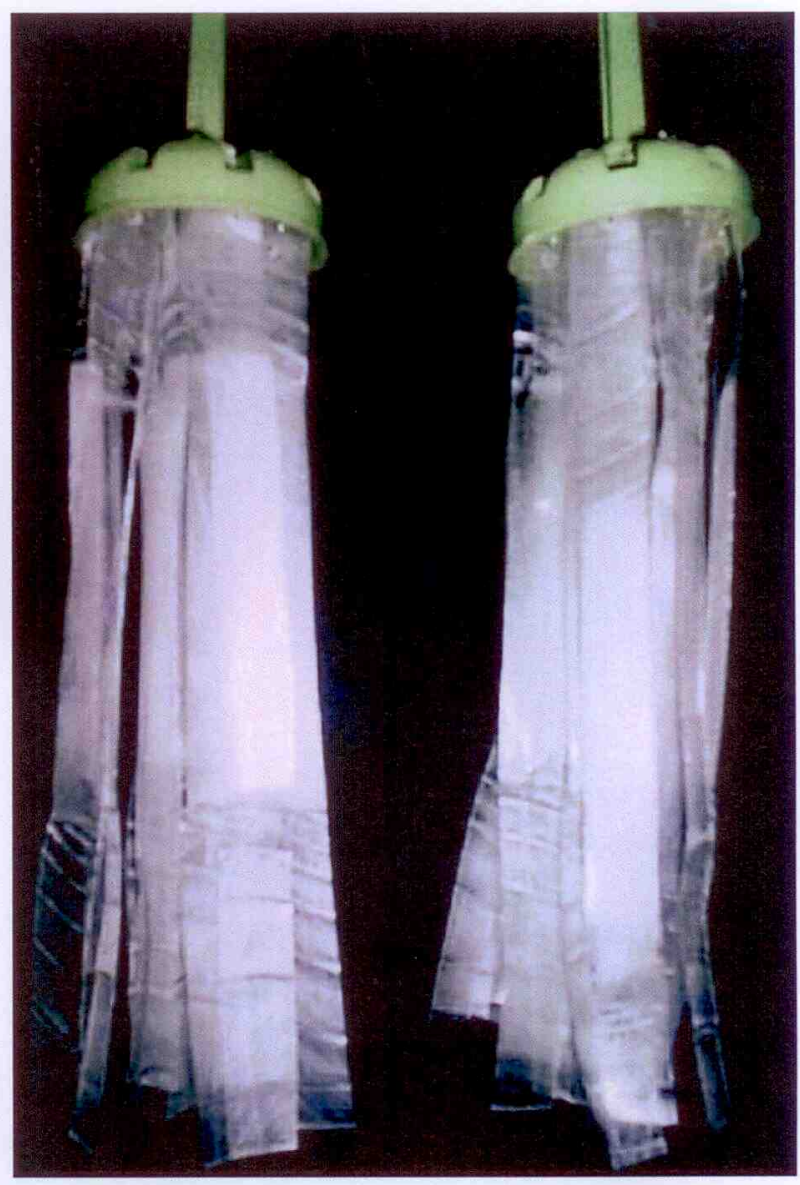

Figura3-cortinas para meio aderido 


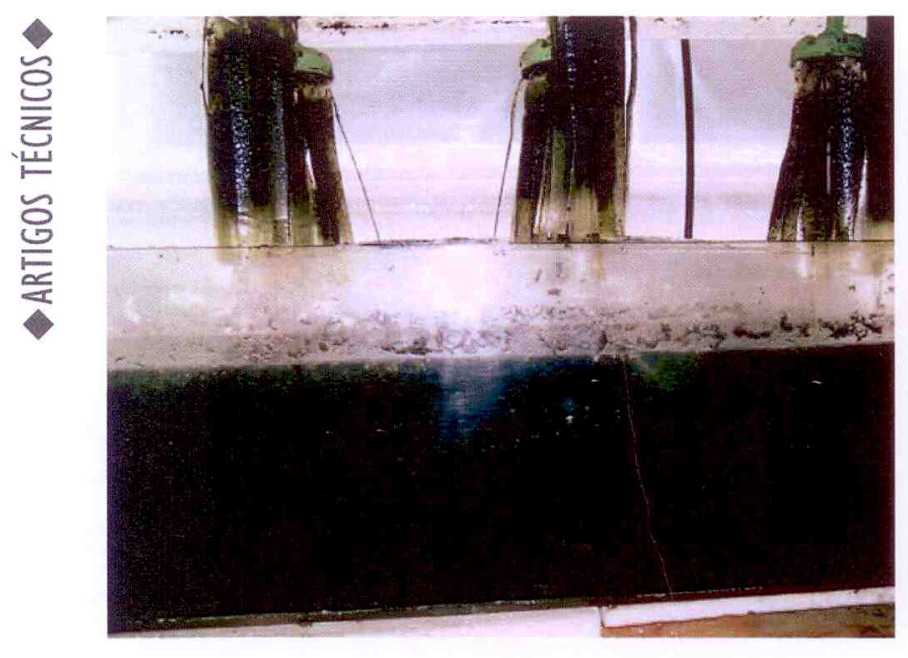

Figura 4- cortinas no tanque séptico durante operação

No estudo realizado por BARBOSA (2006) para avaliar a eficiência de remoção em função do tempo de detenção, o TDH (tempo de detenção hidráulico) de 18 horas apresentou melhores resultados na eficiência de remoção de DQO e DBO. Por esta razão em todos os experimentos $0 \mathrm{TDH}$ adotado foi de 18 horas.

\subsection{A Unidade de Tratamento Tubular de Esgoto}

A estrutura da UTTE foi construída com um tubo de PVC (policloreto de vinila) de $150 \mathrm{~mm}$ de diâmetro e $4 \mathrm{~m}$ de comprimento. A tubulação foi vedada e foram inseridos orifícios nas tampas a 4,5 cm para controlar o nível da massa líquida no interior do tubo. O tubo de PVC foi preenchido com um material suporte para promover o crescimento da biomassa e conseqüentemente o tratamento do efluente. A estrutura foi instalada numa inclinação de $0,28 \mathrm{~m} / \mathrm{m}$ da tubulação. A Figura 6 ilustra a Unidade de Tratamento Tubular de Esgoto em estudo.

Para o material de enchimento foram usadas tiras de garrafa PET lixadas (Fig. 7) do mesmo modo que as cortinas do TS. Foram utilizadas tiras de garrafa PET, pois se apresentaram como um bom material para a aderência de massa microbiológica, de baixo custo e fácil aquisição (ALTVATER, 2005). A tubulação, com volume útil aproximado de 11 litros, operou com um tempo de detenção de aproximadamente 7 horas. Foram inseridas 10 tiras de 4 metros de comprimento e $4,5 \mathrm{~cm}$ de largura, ou seja, 3,6 $\mathrm{m}^{2}$ de área de contato total, $\left(327 \mathrm{~m}^{2} / \mathrm{m}^{3}\right)$.

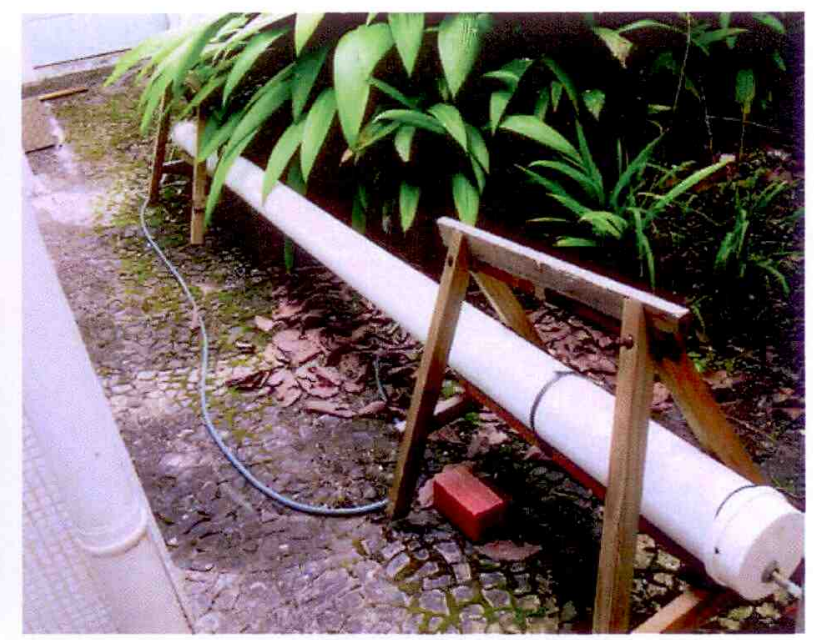

Figura 5- unidade de tratamento tubular de esgoto

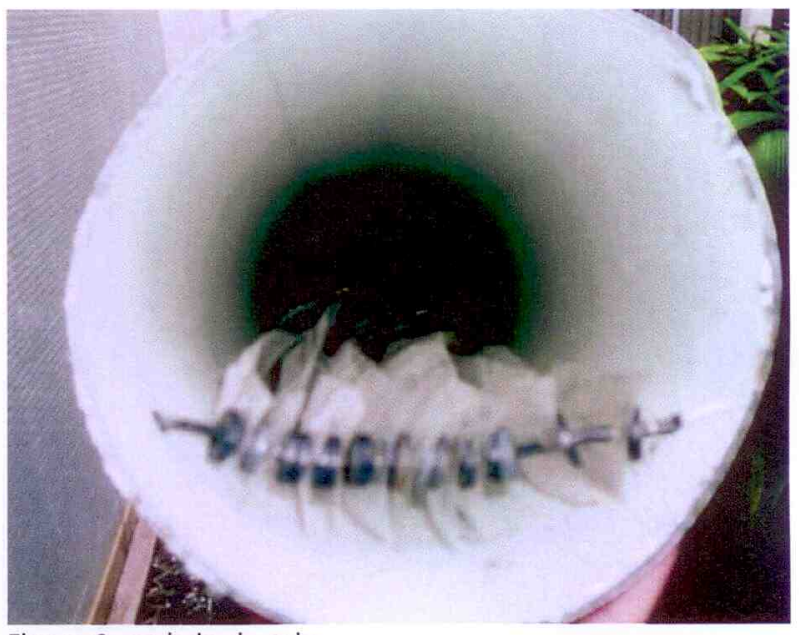

Figura 6 - recheio do tubo

\subsection{Partida e Operação do Sistema}

O TA operou com um volume de $160 \mathrm{~L}$ de esgoto sintético, que era preparado a cada 4 dias. Caso esse volume, do TA, não fosse utilizado pelo sistema durante esse período, o seu excedente era então descartado, sendo adicionada uma nova solução de esgoto sintético. A cada reposição de esgoto, a unidade era lavada com água limpa para evitar o acúmulo de sedimentos no fundo do tanque e a formação de biofilme em suas paredes.

A partida do tanque séptico foi realizada inoculando lodo anaeróbio de reator UASB da ETE Atuba-Sul (Curitiba) em proporção de $30 \%$ do volume útil do TS e alimentado com esgoto sintético. As cortinas do TS foram imersas no mesmo lodo durante 4 semanas e alimentadas com solução de leite em pó para promover o crescimento de biomassa. A UTTE também foi alimentada com lodo anaeróbio diluído e solução de leite em pó durante 4 semanas. O leite em pó foi escolhido por ser rico em nutrientes necessários ao metabolismo microbiano, com nitrogênio e fósforo, além da facilidade em seu preparo.

Após 1 mês o sistema foi disposto na configuração 
ilustrada na Figura 1 e alimentada continuamente com esgoto sintético. O sistema operou desta forma durante 3 meses, para que ocorresse estabilização, até dar-se início à coleta de dados.

A vazão foi de $25 \mathrm{~mL} / \mathrm{min}$ de modo que o tempo de detenção hidráulico do TS fosse igual a 18 horas. A bomba dosadora (BD) utilizada é da marca Milan, modelo BP - 600, com vazão máxima de $80 \mathrm{~mL} / \mathrm{min}$ e vazão mínima de $15 \mathrm{~mL} / \mathrm{min}$ para uma mangueira de silicone com $5 \mathrm{~mm}$ de diâmetro interno. Durante a operação da BD foram realizadas semanalmente aferições da vazão, pois devido ao desgaste da mangueira poderiam ocorrer variações.

\subsection{Monitoramento do Sistema}

Os parâmetros que foram analisados para caracterizar a eficiência do sistema são $\mathrm{DBO}_{5^{\prime}} \mathrm{DQO}_{3^{\prime}} \mathrm{DQO}_{\text {sol }^{\prime}}$ Carbono Orgânico Total (COT), Sólidos Totais, Sólidos Suspensos, Sólidos Dissolvidos e Turbidez. Foram analisados ainda $\mathrm{pH}$, alcalinidade e temperatura.

A DOOsol foi obtida após a filtração em membrana micropore de $0,45 \mu \mathrm{m}$. O COT foi analisado em equipamento analisador de carbono orgânico, da marca Shimadzu, modelo COT - V CPH.

As análises de alcalinidade total e de bicarbonato foram feitas conforme proposta de RIPLEY (1986). O autor ainda cita que a relação alcalinidade de bicarbonatos/alcalinidade total serve de parâmetro de monitoramento do bom funcionamento de reatores anaeróbios. Esta relação deve ser superior a 0,5.

As análises seguiram os métodos analíticos recomendados pelo "Standard Methods" (AWWA; APHA; WEF, 1995).

Os pontos de coleta estão ilustrados na Figura 1, sendo: a entrada do tanque séptico (1), advindo do tanque de armazenamento do esgoto, a saída do tanque séptico (2) que corresponde à entrada do tubo, e a saída do tubo (3), sendo, portanto, 3 pontos de amostragem.

\section{3 - RESULTADOS E DISCUSSÃO}

O sistema TS-UTTE operou durante 107 dias, durante os quais foram avaliados diversos parâmetros físico-químicos. As tabelas 2 e 3 apresentam os resultados das análises para $\mathrm{pH}$, matéria orgânica, turbidez e sólidos para os 3 pontos de coleta. Não foram apresentados os demais parâmetros, pois foram avaliados apenas para acompanhamento do sistema ou foram realizadas poucas amostragens, não compondo um número suficiente para uma avaliação estatística adequada.

As tabelas são infelizes para visualização dos resultados. Desta forma será analisado o tratamento isolado de cada unidade (TS e UTTE) e depois o conjunto TS-UTTE.

As Figuras 7 e 8 ilustram o valor da DQO e dos SS à montante e à jusante do TS ao longo das amostragens, bem como as respectivas eficiências de remoção. A Figura 9 condensa as informações a respeito das eficiências alcançadas pelo TS com respeito à $\mathrm{DBO}$, DQO, SS e ST. As médias foram respectivamente 37,5 $\%, 46,6 \%, 62,3 \%$ e 20,4\%, excluindo-se alguns poucos dados que apresentaram eficiências negativas.

Como esperado, o TS apresentou eficiências moderadas de DBO e DQO, e eficiências razoáveis em termos de SS, a qual é a característica principal dos tanques sépticos. A eficiência de remoção de DOO para o TS operando nas mesmas condições sem cortinas para meio aderido foi de 42,0\% (ALTVATER, 2008), de modo que se observou pouca melhoria de eficiência com a modificação proposta, a qual apresentou eficiência média de 46,6\%.

O comportamento isolado da UTTE é observado nas Figuras 10 e 11 em termos de DOO e SS. Assim como nos dados do TS observa-se um grande amplitude de valores, cuja origem foram as condições insuficientes de homogeneização no TA que propiciava a sedimentação e a variação da temperatura ambiente, e consequentemente da massa líquida visto que o sistema está exposto no ambiente. Apesar da amplitude de variação da temperatura no sistema ser menor do que no ambiente, esta interfere fortemente nos processos biológicos.

A Figura 12 apresenta os dados de eficiência de remoção de DBO, DQO, SS e ST para a UTTE. As médias foram respectivamente $59,9 \%, 48,7 \%, 45,2 \%$ e $12,1 \%$. A UTTE atua como uma unidade de pós-tratamento, realizando o polimento da matéria orgânica dissolvida. As remoções de sólidos são mais modestas, porém, apesar de não ser o objetivo do sistema, promoveu uma remoção adicional de SS efluentes do TS.

Da mesma forma que apresentado anteriormente, as Figuras 13 e 14 ilustram os dados de entrada e saída da DQO e SS, agora sob a visão do sistema completo TS-UTTE. As variações também foram grandes, exigindo uma análise estatística. A Figura 15 apresenta esta análise em termos de eficiência para os parâmetros DBO, DOO, SS e ST. O sistema TS-UTTE apresentou eficiências médias dos respectivos parâmetros iguais a $66,2 \%, 70,3 \%, 73,4 \%$ e $20,7 \%$. Considerando a eficiência média de tratamento do TS, a carga orgânica aplicada no UTTE foi de $0,88 \mathrm{~kg} \mathrm{DQO} / \mathrm{m}^{3}$.dia.

A relação entre alcalinidade devida a bicarbonatos e alcalinidade total variou entre 0,51 e 0,66 no efluente do TS e entre 0,62 e 0,70 no efluente da UTTE. Esta relação, que serve como parâmetro de monitoramento de sistemas anaeróbios, manteve-se sempre acima do valor 0,5, recomendado à boa operação do sistema.

A eficiência global em termos de DBO e DOO do sistema foi muito boa, comparável a outros sistemas combinados de tanque séptico seguido de pós-tratamento anaeróbio. As médias de remoção da matéria 
orgânica foram muito próximas às encontradas por PHILIPPI et al. (1999), avaliando um sistema composto por tanque séptico seguido de um tratamento por zona de raízes, e encontrando eficiências médias de 71 e $69 \%$ para DQO e DBO.

O destaque do sistema TS-UTTE se deve principalmente ao fato de ser composto de unidades econômicas, de simplicidade construtiva e operacional. As uni- dades se complementam em suas limitações, a UTTE realiza um polimento da matéria orgânica pouco removida no TS e este realiza um tratamento primário removendo principalmente SS de modo a não obstruir a UTTE. Inesperadamente a UTTE apresentou uma razoável eficiência de remoção de SS, melhorando ainda mais a qualidade do efluente.

\begin{tabular}{|c|ccc|ccc|ccc|}
\cline { 2 - 9 } Dia & ES & TS & UTTE & ES & TS & UTTE & ES & TS & UTTE \\
\hline 15/set & n.m. & n.m. & & 440 & 168 & 97 & 191 & 73 & 36 \\
20/set & 6,7 & 6,8 & 6,8 & 491 & 233 & 188 & n.m. & n.m. & n.m. \\
22/set & n.m. & n.m. & & 762 & 310 & 154 & n.m. & n.m. & n.m. \\
25/set & 7,0 & 6,9 & 6,9 & 383 & 286 & 191 & n.m. & n.m. & n.m. \\
28/set & 6,8 & 6,7 & 6,7 & 415 & 369 & 102 & 182 & 180 & 40 \\
02/out & 6,5 & 6,9 & 6,9 & 479 & 242 & 225 & n.m. & n.m. & n.m. \\
04/out & 6,7 & 6,7 & 6,7 & 268 & 130 & 102 & 107 & 61 & 34 \\
11/out & 6,9 & 7,0 & 7 & 350 & 228 & 146 & 165 & 127 & 71 \\
18/out & 6,7 & $n . m$. & n.m. & 481 & 176 & n.m. & n.m. & n.m. & n.m. \\
24/out & 7,4 & 7,0 & 7 & 379 & 391 & 267 & 156 & 237 & 155 \\
17/nov & 6,8 & 7,1 & 7,1 & 616 & 137 & 46 & n.m. & n.m. & n.m. \\
23/nov & n.m. & n.m. & n.m. & 422 & 137 & 11,4 & n.m. & n.m. & n.m. \\
30/nov & n.m. & n.m. & n.m. & 638 & 239 & 91 & n.m. & n.m. & n.m. \\
13/dez & 6,7 & 7,0 & 7,0 & 547 & 342 & 79,8 & n.m. & n.m. & n.m. \\
15/dez & 7,0 & 6,9 & 6,9 & 562 & 443 & 145 & 360 & 303 & 37 \\
20/dez & 6,9 & 9,9 & 9,9 & 826 & 494 & 236 & 434 & 244 & 48 \\
\hline
\end{tabular}

Tabela 2 - Resultados de alcalinidade e matéria orgância

ES - Esgoto Sintético de alimentação do Tanque Séptico

\begin{tabular}{|c|c|c|c|c|c|c|c|c|c|}
\hline \multirow{2}{*}{ Dia } & \multicolumn{3}{|c|}{ Turbidez (NTU) } & \multicolumn{3}{|c|}{$\begin{array}{l}\text { Sólido Suspensos } \\
(\mathrm{mg} / \mathrm{L})\end{array}$} & \multicolumn{3}{|c|}{ Sólidos Totais (mg/L) } \\
\hline & ES & TS & UTTE & ES & TS & UTTE & ES & TS & UTTE \\
\hline $15 /$ set & 158 & 7,4 & 8,3 & 260 & 70 & 24 & 1242 & 848 & 760 \\
\hline $20 /$ set & 26,1 & 6,3 & 5,5 & 90 & 28 & 16 & 724 & 660 & 684 \\
\hline $22 /$ set & 85,5 & 11,7 & 14,2 & 150 & 68 & 84 & 1012 & 756 & 766 \\
\hline $25 /$ set & 34,5 & 24,6 & 9,4 & 84 & 20 & 8 & 846 & 738 & 666 \\
\hline $28 /$ set & 42 & 35 & 6,3 & 178 & 74 & 26 & 924 & 978 & 696 \\
\hline 02/out & 69 & 16,8 & 15,5 & 200 & 42 & 34 & 952 & 740 & 804 \\
\hline 04/out & 29,8 & 24,1 & 9,9 & 128 & 60 & 28 & 984 & 908 & 862 \\
\hline 11/out & 18,2 & 7,5 & 6,0 & 72 & 28 & 10 & 858 & 530 & 594 \\
\hline 18/out & 76,5 & 12,7 & $\mathrm{n} . \mathrm{m}$ & n.m & n.m & n.m & $\mathrm{n} \cdot \mathrm{m}$ & n.m & n.m \\
\hline 24/out & 67 & 15,7 & 13,5 & 80 & 28 & 20 & 564 & 796 & 632 \\
\hline $17 /$ nov & 159 & 9,9 & 7,4 & 180 & 66 & 48 & 1312 & 928 & 914 \\
\hline $23 /$ nov & 77 & 13,8 & 7,7 & 178 & 102 & 60 & 1086 & 886 & 876 \\
\hline $30 /$ nov & $\mathrm{n} . \mathrm{m}$. & n.m. & n.m & n.m. & $\mathrm{n} . \mathrm{m}$. & $\mathrm{n} \cdot \mathrm{m}$. & n.m. & n.m. & n.m. \\
\hline $13 / \mathrm{dez}$ & 63 & 30,8 & 11,4 & 232 & 110 & 62 & 950 & 962 & 774 \\
\hline $15 / \mathrm{dez}$ & 60 & 24,8 & 27,5 & 70 & 76 & 52 & 988 & 894 & 922 \\
\hline $20 / \mathrm{dez}$ & 89 & 18,7 & 6,5 & n.m. & n.m. & n.m. & n.m. & n.m. & n.m. \\
\hline
\end{tabular}

Tabela3 - Resultados de turbidez e sólidos

ES - Esgoto Sintético de alimentação do Tanque Séptico

UTTE - Efluente de saída da Unidade de Tratamento de Esgoto 


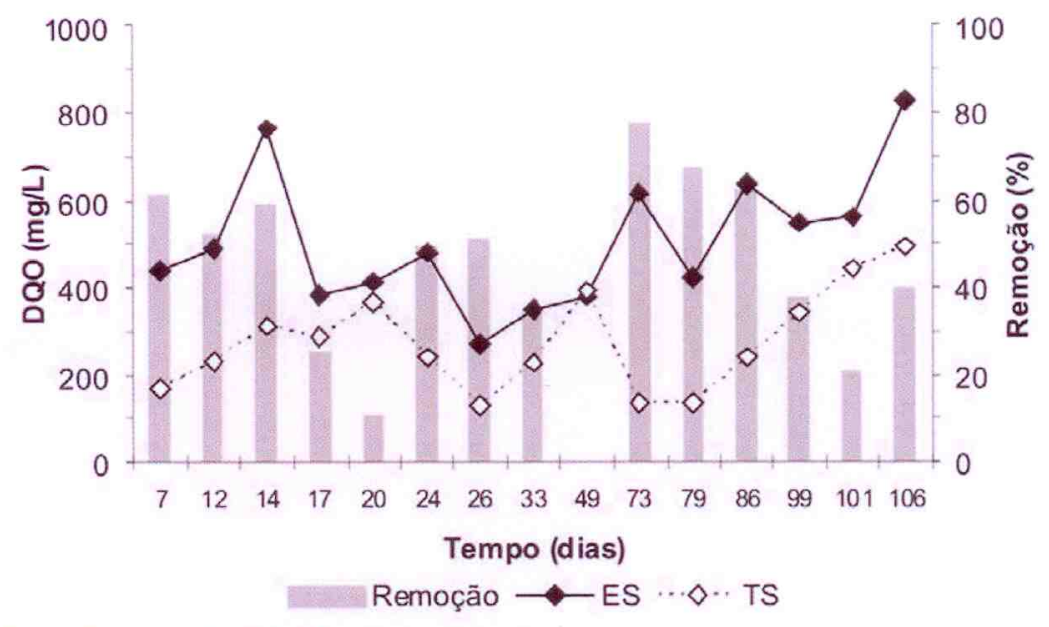

Figura 7 - remoção de DQO - tanque - séptico

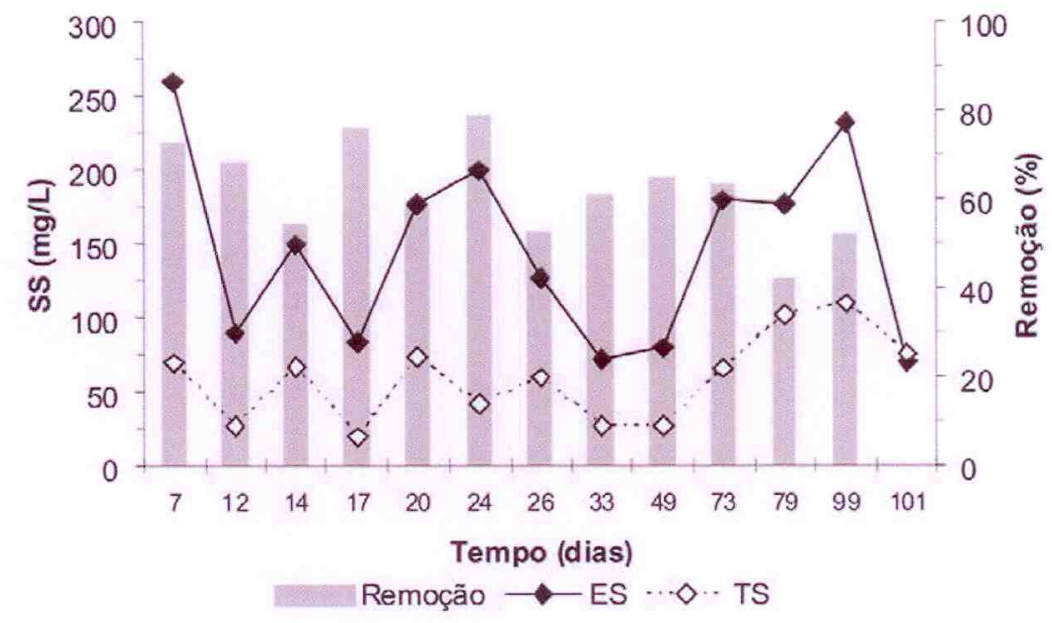

Figura 8 -remoção de SS - tanque séptico

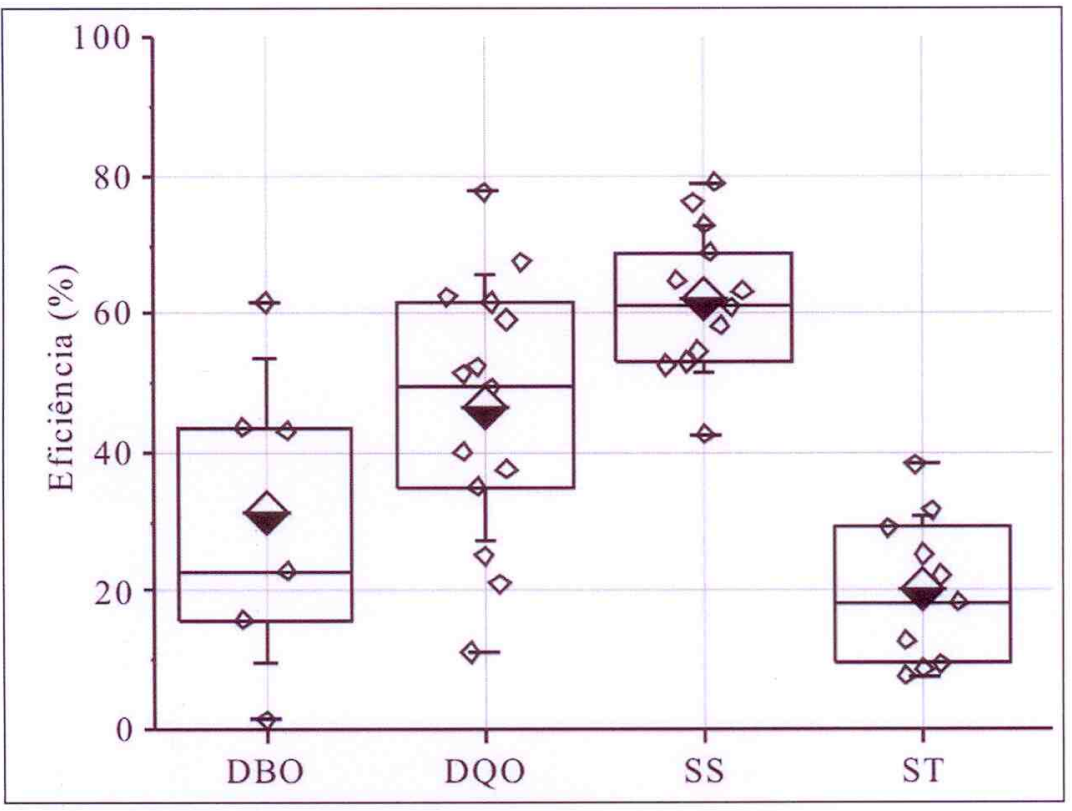

Figura 9 - eficiência de remoção de DBO, DQO, SS e ST - tanque séptico 
号

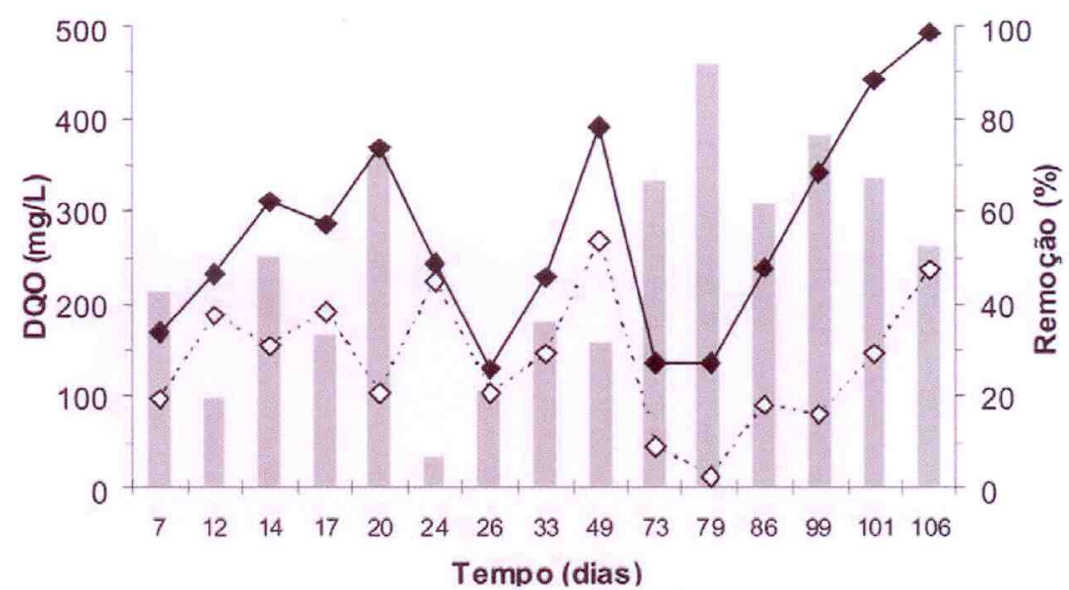

Remoção $\longrightarrow$ TS $\cdots \diamond \cdot$ UTTE

Figura 10 - remoção de DQO - UTTE

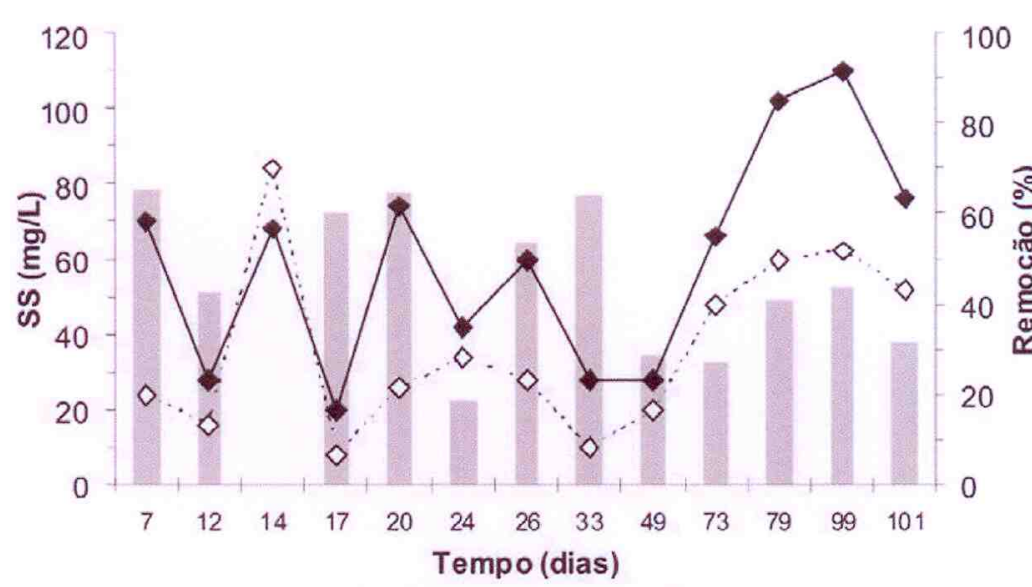

Remoção $\bullet$ TS $\cdots \diamond \cdots$ UTTE

Figura 11 -remoção de SS - UTTE

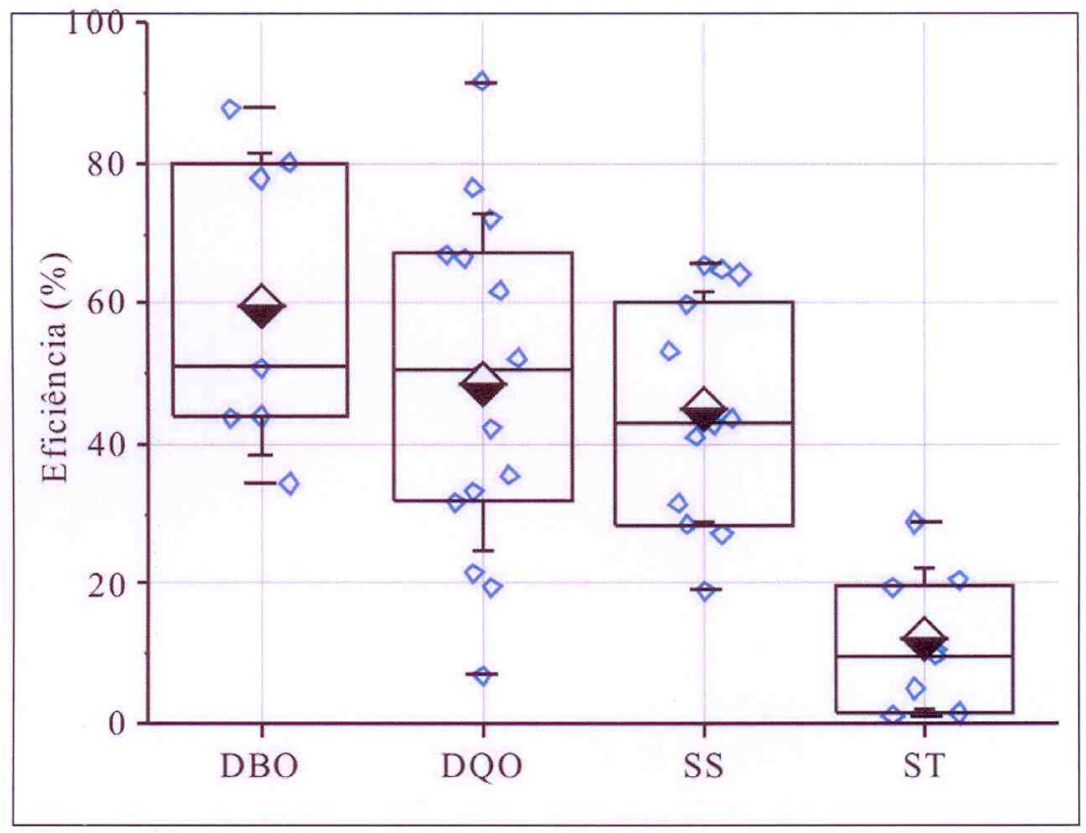

Figura 12 - eficiência de remoção de DBO, DQO, SS e ST - UTTE 


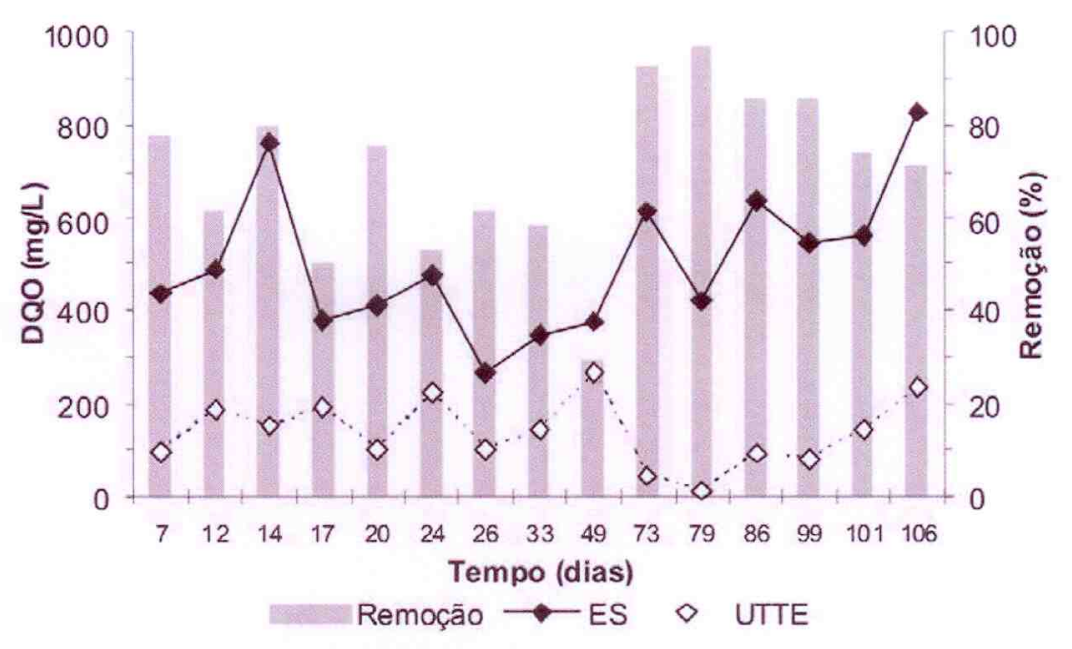

Figura 13 - remoção de DQO - sistema TS-UTTE

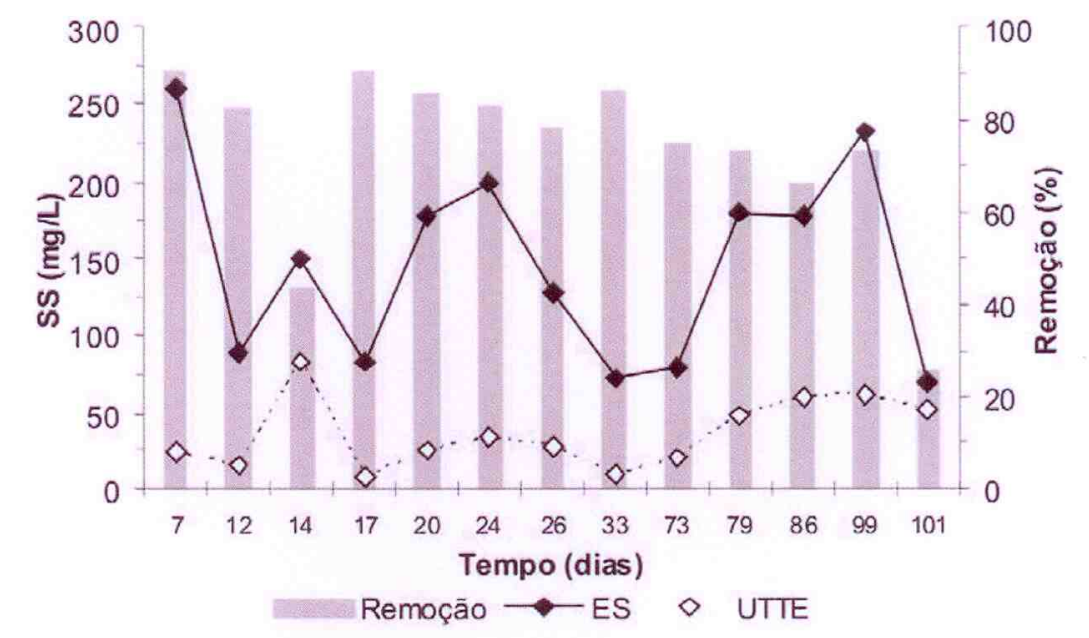

Figura 14 - remoção de SS - sistema TS-UTTE

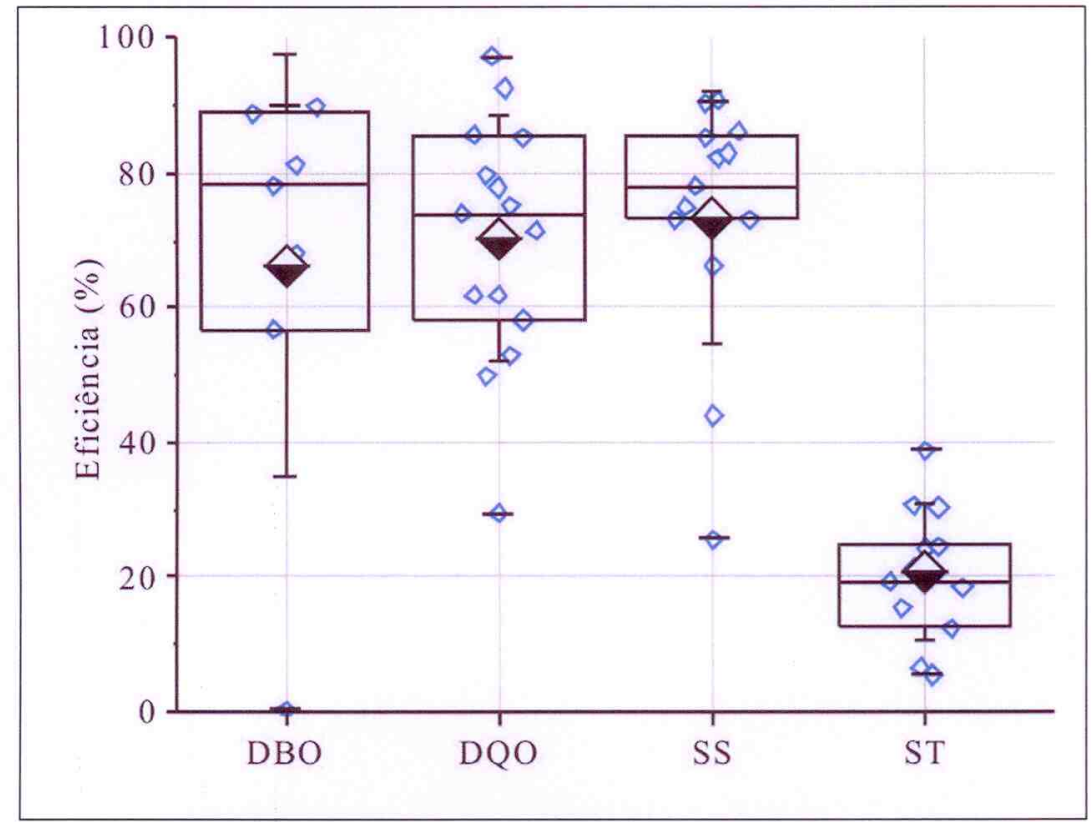

Figura 15 - eficiência de remoção de DBO, DQO, SS E ST - sistema TS-UTTE 


\section{4- CONCLUSÕES}

Esta pesquisa teve como objetivo investigar o desempenho de um sistema simples e de baixo custo para servir como alternativa a sistemas de tratamento de esgoto em locais desprovidos de coleta. A estratégia foi procurar aumentar a eficiência de tratamento de um sistema clássico, o Tanque Séptico, por meio da adição de cortinas que promoveram o crescimento de biomassa e consequentemente a melhoria do tratamento do despejo no interior do TS. Outra abordagem foi a proposta de um novo conceito de pós-tratamento denominado Unidade Tubular de Tratamento de Esgoto (UTTE), constituída de uma tubulação convencional de PVC em cujo interior foram dispostas tiras de material de garrafas PET previamente lixadas as quais serviram de meio suporte para desenvolvimento de biomassa.

A adoção das tiras de garrafas PET como material de suporte para biomassa no TS e na UTTE se deu devido a resultados já comprovados de viabilidade técnica, econômica e também sob aspecto ecológico, reaproveitando um material que poderia ser descartado.

Os resultados apontam que a proposta de adição de cortinas no TS não rendeu os resultados esperados, promovendo uma melhoria do tratamento, porém não significativa. A eficiência média de remoção de DOO configuração convencional.

A UTTE apresentou eficiência média de remoção de $\mathrm{DBO}, \mathrm{DQO}$, SS de $59,9 \%, 48,7 \%, 45,2 \%$, respectivamente. Enquanto que o sistema combinado TS-UTTE apresentou para os mesmos parâmetros eficiências de $66,2 \%, 70,3 \%, 73,4 \%$, respectivamente. Estes resultados apontam o sistema combinado como uma boa alternativa para tratamento de baixo custo para regiões desprovidas de coleta. Todavia, recomenda-se tratamento primário para o afluente a este sistema, composto de gradeamento e desarenador, visto que o efluente simulado não contém sólidos grosseiros e areia, materiais estes que podem comprometer o desempenho do sistema, principalmente da UTTE.

Os resultados deste trabalho, apesar de possuir um caráter inovador e comprovar o conceito da UTTE, conduzem também a uma autocrítica e sugestões. Primeiramente um melhor controle das condições de entrada a fim de reduzir as variações nas concentrações do afluente ao sistema. A utilização de esgoto doméstico real como despejo para avaliar o desempenho do sistema. E ainda o ensaio com outros materiais de recheio, com maior rugosidade e área de contato, a fim de proporcionar uma maior capacidade de fixação de biofilme para o tratamento.

\section{AGRADECIMENTOS \\ À Fundação Nacional da Saúde, Funasa, pelo apoio e financiamento à pesquisa.}

\section{BIBLIOGRAFIA}

ABNT. Construção e instalação de fossas sépticas e disposição dos efluentes finais. NBR 7229, São Paulo/ SP, 1993.

ANDRADE NETO, C. O. Sistemas simples para tratamento de esgotos sanitários. Rio de janeiro, ABES, 1993.

ALTVATER, P. K. Estudo da Potencialidade de Tratamento de um Filtro Biológico de Fluxo Horizontal em Rede de Drenagem Urbana. Curitiba, 2005. Trabalho de Conclusão do Curso de Engenharia Ambiental, Setor de Tecnologia, Universidade Federal do Paraná.

ALTVATER, P. K. Avaliação do Desempenho de um Tanque Séptico Modificado e Tratamento Complementar. Curitiba, 2008. Dissertação (Mestrado em Engenharia de Recursos Hídricos e Ambiental) - Setor de Tecnologia, Universidade Federal do Paraná.

AWWA, APHA and WEF. Standard methods for the examination of water and wastewater. 18. ed., Washington, 1995.

BARBOSA, S. A. Avaliação de biofiltro aerado submerso no pós-tratamento do efluente de tanque séptico. Curitiba, 2006. 228 f. Dissertação (Mestrado em Engenharia de Recursos Hídricos e Ambiental) - Setor de Tecnologia, Universidade Federal do Paraná.

INSTITUTO BRASILEIRO DE GEOGRAFIA E ESTATíSTICA. Pesquisa Nacional de Saneamento Básico 2000. Rio de Janeiro, 2002.

PHILIPPI, L. S.; COSTA, R. H. R.; SEZERINO, P. H. Domestic effluent treatment through integrated system of septic tank and root zone. Water Science and Technology, Inglaterra, v. 40, n. 3, p. 125-131, 1999.

RIPLEY, L. E.; BOYLE, W. C.; CONVERSE, J. C. Improved alkalimetric monitoring for anaerobic digestion of high-strength wastes. Journal of the Water Pollution Control Federation, v. 58, n 5, p. 406-411, 1986.

SNIS. Sistema Nacional de Informações sobre Saneamento. Diagnóstico dos Serviços de Água e Esgoto. Ministério das Cidades. Brasília, 2003

.TORRES, P. Desempenho de um Reator Anaeróbio de Manta de Lodo (UASB) de Bancada no Tratamento de Substrato Sintético Simulando Esgoto Sanitário. São Carlos. 1992. Dissertação (Mestrado) - Escola de Engenharia de São Carlos - Universidade de São Paulo. 